Tropical Journal of Pharmaceutical Research, June 2005; 4 (1): 331-339

(c) Pharmacotherapy Group, Faculty of Pharmacy, University of Benin Benin City, Nigeria.

All rights reserved.

Research Article

Available online at http://www.tjpr.freehosting.net

\title{
Comparative evaluation of selected starches as adsorbent for Thin-layer Chromatography
}

\author{
TA Abere, HA Okeri and LO Okafor
}

Faculty of Pharmacy, University of Benin, PMB 1154, Benin City, Nigeria

\begin{abstract}
Purpose: A variety of tested organic and inorganic adsorbents are available today specifically for thin-layer chromatography. The most commonly used is silica gel which is an inorganic adsorbent. Organic substances like cellulose, polyethylene are also used. All these are imported into Nigeria and are unhealthy for economic policies. Most commonly used adsorbent may not be easy to produce locally, but starch, which is a very common product, can be made very readily available.

Method: Comparative tests were carried out on cassava, guinea corn and irish potato starches to evaluate and determine suitability as adsorbents for thin-layer chromatography. The starches were used in their natural forms and various modified forms:-formamide, paraffin-impregnated forms and derivatized forms so as to exhibit different properties using different solvent systems to separate different classes of compounds namely alkaloids, amine acids, lipids and steroids with silica gel as standard.

Results: The results obtained have proved starch to be a suitable adsorbent both in its naturally occurring and modified forms. Good separations of amino acids and steroids were obtained on natural layers when compared with silica gel, while alkaloids on formamideimpregnated layers and lipids on paraffin-impregnated layers also gave encouraging results. The acetylated starch by suitable modification should produce good results.

Conclusion: The suitability of natural starches and its modifications as adsorbents for TLC has thus been established and seems very promising for future use.
\end{abstract}

Key words: Starch, cassava, guinea corn, Irish potato, adsorbent, thin-layer chromatography.

*Corresponding Author, Tel:+2348023395616 E-mail: abere@uniben.edu, eseabere@yahoo.com. 


\section{Abere et al}

\section{Introduction}

Starches are very popular in the pharmaceutical industry in which they are used as binders and disintergrants in tableting. Several nonpharmaceutical starches have been investigated as tableting excipients with positive results. (1-6) Many stages in the preparation of pharmaceutical products ranging from the detection and isolation of trace amounts of naturally occurring or synthetic compounds to the large scale production of medicinal agents require some form of separation and purification. ${ }^{(7)}$ Chromatography is a term which refers to a number of highly efficient separation techniques based on one of a number of principles: adsorption, partition,ion-exchange or exclusion. The separation of a mixture of substances is effected by differential distribution of the substances between two phases, one of which is percolated through the other.

Chromatography has very wide applications, both for analytical and preparative purposes. It has been used to resolve mixtures, to isolate constituents of mixtures, to monitor the progress of chemical reaction by checking for reactions and/or products at intervals to access the purity of compounds-a pure compound shows as a single spot or peak while impure compound results in the appearance of many spots or peaks. Chromatography is also used to compare substances by the use of certain values referred to as $R_{\mathrm{f}}$-values ${ }^{(8)}$.

Different adsorbents have been used as the stationary phase in thin-layer chromatography either in their natural or in modified forms for the separation of a wide variety of substances. The most commonly used is silica gel which is an inorganic adsorbent. Organic substances like cellulose, polyethylene are also used. All these are imported into Nigeria, which is unhealthy for the economic policies of the present administration. Most of the commonly used adsorbents may not be easy to produce locally, but starch, which is a very common product can be made readily available, hence this research aimed at investigating the suitability of starches as adsorbents for thin-layer chromatography.

\section{Experimental}

\section{Extraction of Starches}

Cassava, Guinea corn and Irish potato starches were prepared in our laboratory from Manihot utilissima, Sorghum vulgare and Solanum tuberosum respectively.

Starch was extracted from the tubers of Manihot utilissima and Solanum tuberosum and seeds of Sorghum vulgare which were washed, dried in air, milled, then reduced to fine pulps using a wet blender(Moulinex, France). The wet mass was transferred into a clean muslin bag and blended in a bucket of water. The starch was extracted by squeezing and rubbing the bag continuously. The suspension of starch was allowed to settle. The supernatant was decanted and more water was used to wash the starch. After decanting the supernatant, the starch sediment was air -dried before being oven-dried(Kotterman,Germany) at $40^{\circ} \mathrm{C}$ for $6 \mathrm{~h}$. The starch was bleached with sodium hypochlorite at $\mathrm{pH} 10.0(3020 \mathrm{pH}$ meter,Jenway,UK) and $30^{\circ} \mathrm{C}$ for $10 \mathrm{~h}$. After bleaching, the slurry was strained through a fine muslin bag to recover the starch. The starch was washed several times with water until no trace of the oxidant was found in the effluent and the $\mathrm{pH}$ about 7.0.The wet starch was finally washed with $95 \%$ ethanol and strained. The starch was ovendried at $50{ }^{\circ} \mathrm{C}$ for $18 \mathrm{~h}$, and the dried mass forced through a $0.125 \mathrm{~mm}$ aperture sieve. The treatment with ethanol was to facilitate drying.

\section{Preparation of the Thin-layers}

The starches were used in different forms to prepare the plates, i.e.

--- In the original form

--- Impregnation with formamide

These were carried out by dipping the airdried plates in a mixture of $80 \mathrm{ml}$ acetone and $20 \mathrm{ml}$ formamide $(4: 1)$ in a chromatank. ${ }^{(9)}$. The mixture rises up through the plates.

---impregnation with liquid paraffin.

A $50 \%$ solution of liquid paraffin in light petroleum $^{(9)}$ rises up through the plates which 
were exposed to air to allow excess light petroleum to evaporate.

--- As the acetylated form-starch acetate.

$30 \mathrm{~g}$ of starch was added to $50 \mathrm{ml}$ of acetic acid and $100 \mathrm{ml}$ acetic anhydride. $5 \mathrm{ml}$ concentrated sulphuric acid was added, $1 \mathrm{ml}$ at intervals to prevent violent reaction and charring. The mixture stirred for two days for acetylating to complete.

\section{Sample Solutions}

The samples (powders and liquids) were made into solutions of $1 \%$ before spotting. The solvents used were:

(a) Chloroform (reserpine, papaverine, quinidine, oleic acid, palmitic acid, cod-liver oil, castor oil)

(b) Distilled water (alanine, glycerine, methionine, histidine, scopolamine, atropine, strychnine)

(c) $70 \%$ ethanol (ouabain, quinine, digoxin, digitoxin)

(d) Acetic acid (cinchonine)

\section{Solvent Systems}

(a) Plain starch plates.

(i) Solvent systems for alkaloids were:

Petroleum ether: chloroform 70:30

Benzene; acetone: chloroform 20:30:50

Acetone: chloroform 40:60

Pentane: diethylamine $80: 20$

Methanol: chloroform 40:60

(ii) Solvent systems for steroids were:

Chloroform: petroleum ether 50: 50

Chloroform: petroleum ether 60: 40

N-butanol: acetic acid: water 50:40:10

N-butanol: acetic acid: water 50:20:30

N-butanol: acetic acid 90:10

Chloroform: ethanol 80:20

Chloroform: ethanol 90:10

Chloroform: acetone: ethanol 50:40: 10

(iii) Solvent systems for amine acids were:

Chloroform: petroleum ether 50: 50

N-butanol: acetic acid: water 60:30:10

N-butanol: acetic acid: water 60:15:25

N-butanol: acetone: diethylamine:

water 35:35:10:20

(iv) Solvent systems for fatty acids and fixed oils were:

Petroleum ether: chloroform 40:60

Chloroform: petroleum ether: acetone 60:30:10

(a)

Paraffin impregnated plates

Solvent systems for fatty acids and fixed oils.

N-butanol: acetic acid 60:40

Chloroform; ethanol 90:10

(c) Formamide impregnated plates

Solvent systems for alkaloids.

Pentane: benzene; chloroform

35:45:20

Pentane: benzene 45:55

Pentane; ethylacetate: diethylamine 50:45:5

Diethylamine; pentane 80: 20

Chloroform: petroleum ether 30:70

(d) Acetylated plates

(i) Solvent system for fatty acids and fixed oils

Petroleum ether: diethylether: acetic acid 60:30:10

(ii) Solvent systems for alkaloids

Pentane: diethylamine 80:20

Acetone: chloroform 90:10

Ethanol: chloroform 10:90

Development was by the ascending onedimensional method. Samples resolved on the plates were visualized using either general or specific methods: thus ultraviolet light indicated fluorescent samples (examined in both long-(c.365nm) and short(c.263nm) wavelength ultraviolet light). Dragendorff's reagent was used in the form of spray for the general detection of alkaloids, Ninhydrine for amino acids, anisaldehyde in sulphuric acid for steroids (require the sprayed chromatogram to be 
heated at $100{ }^{\circ} \mathrm{C}$ for varying times (5-20 $\mathrm{min}$ ) in order to develop the colours), iodine tank for fatty acids, fixed oils and alkaloids. After detection, the spots were recorded, photocopied and the rate of advance of each in the solvent systems calculated by determination of $\mathrm{R}_{\mathrm{f}^{-}}$and $\mathrm{hR}_{\mathrm{f}^{-}}$values.

$$
\mathbf{R}_{\mathbf{f}^{-}}=\frac{\text { Distance moved by the substance }}{\text { Distance moved by the solvent }}
$$

$$
H_{\mathbf{f}^{-}}=\mathrm{R}_{\mathrm{f}^{-}} \times 100 .
$$

\section{Results}

Table 1 shows the $\mathrm{HR}_{\mathrm{f}^{-}}$vales of four (4) Amino acids on plain starch layers with silica gel as standard, using five (5) solvent systems. Whereas the samples remained at the origin
Table 2 shows the $\mathrm{HR}_{\mathrm{f}^{-}}$values of three (3) steroids on plain starch layers using various solvent systems. While non-polar solvent systems were able to separate Diosgenin, Digtoxin and ouabain were better separated by polar solvent systems. Chloroform: ethanol 90: 10 separated all three steroids. With Anisaldehyde in sulphuric acid spray reagent, the spots were either pink or purple in colour.

Table 3 shows the $\mathrm{HR}_{\mathrm{f}}$ - values of two (2) fixed oils on plain and paraffin impregnated starch layers using both polar and non-polar solvent systems. With polar solvent systems, Castor oil and Cod liver oil did not move from the origin, however they moved to the solvent front with non-polar solvent systems except in Guinea corn

\begin{tabular}{|c|c|c|c|c|c|}
\hline & & Cassava & Irish potato & Guinea corn & Silica gel \\
\hline Solvent systems & Spots & $R_{f}(x 100)$ & $R_{f}(x 100)$ & $(x 100)$ & $(\times 100)$ \\
\hline Petroleum ether: & Alanine & No movement & No movement & No movement & No movement \\
\hline chloroform 50: 50 & Methionine & No movement & No movement & No movement & No movement \\
\hline & Histidine & No movement & No movement & No movement & No movement \\
\hline & Glycerin & No movement & No movement & No movement & No movement \\
\hline n-butanol:acetic & Alanine & 19 & 22 & 15 & 22 \\
\hline acid:water & Methionine & 34 & 38 & 34 & 35 \\
\hline $60: 30: 10$ & Histidine & 06 & 08 & 03 & 05 \\
\hline & Glycerin & 07 & 10 & 07 & 18 \\
\hline n-butanol:acetic & Alanine & 17 & 20 & 30 & 25 \\
\hline acid:water & Methionine & 42 & 42 & 44 & 47 \\
\hline $60: 15: 25$ & Histidine & 13 & 10 & 13 & 12 \\
\hline & Glycerin & 11 & 15 & 15 & 15 \\
\hline n-butanol:acetone: & Alanine & 18 & 15 & 20 & 22 \\
\hline diethylamine:water & Methionine & 32 & 36 & 30 & 36 \\
\hline $35: 35: 10: 20$ & Histidine & 03 & 08 & 06 & 08 \\
\hline & Glycerin & 09 & 13 & 10 & 20 \\
\hline
\end{tabular}
starch layers when Chloroform : petroleum ether

TABLE 1: $\mathrm{HR}_{\mathrm{f}}$ values of 4 Amino acids on plain starch layers with Silica gel as standard.

when non-polar solvent systems were used as the mobile phase, they separated out in all the starch layers including the standard (silica gel) when polar solvent systems were used. The spots on spraying with Ninhydrine solution were either reddish violet or grayish violet in colour.
65 :35 was used. The fixed oils were well separated on paraffin impregnated starch layers. In lodine tank, the spots were reddish brown in colour. 
Table 4 shows the $\mathrm{HR}_{\mathrm{f}^{-}}$values of alkaloids on plain, formamide impregnated and acetate starch layers with silica gel as standard, using both polar and non-polar solvent systems. When developed on plain starch layers with a nonpolar solvent (Petroleum ether: chloroform 70:30) quinine and papaverine moved while developed with Pentane: benzene: chloroform 35: 45: 20 , strychnine and papaverine moved to the solvent front while quinine and quinidine remained near the origin. With Pentane: diethylamine 80: 20 , only quinine and quinidine moved on Cassava and Irish potato layers, but all the alkaloids -quinine, quinidine, strychnine

TABLE 2: HR $_{f}$ values of 2 Steroids on plain starch layers with Silica gel as standard.

\begin{tabular}{|c|c|c|c|c|c|}
\hline & & Cassava & Irish potato & Guinea corn & Silica gel \\
\hline Solvent systems & Spots & $\mathbf{R}_{\mathbf{f}}(\mathbf{x} 100)$ & $R_{f}(x 100)$ & $\mathbf{R}_{\mathbf{f}}(\times 100)$ & $R_{f} \quad(x 100)$ \\
\hline \multirow{3}{*}{$\begin{array}{l}\text { Chloroform: } \\
\text { petroleum ether } \\
50: 50\end{array}$} & Digitoxin & No movement & No movement & No movement & 68 \\
\hline & Ouabain & No movement & No movement & No movement & 46 \\
\hline & Diosgenin & Moved to SF & Moved to SF & Moved to SF & 58 \\
\hline \multirow{3}{*}{$\begin{array}{l}\text { Chloroform: } \\
\text { petroleum ether } \\
60: 40\end{array}$} & Digitoxin & No movement & No movement & No movement & 65 \\
\hline & Ouabain & No movement & No movement & No movement & 41 \\
\hline & Diosgenin & Moved to SF & Moved to SF & Moved to SF & 48 \\
\hline \multirow{2}{*}{$\begin{array}{l}\text { n-butanol:acetic } \\
\text { acid:water }\end{array}$} & Digitoxin & 91 & 61 & Moved to SF & 62 \\
\hline & Ouabain & No movement & 21 & No movement & 29 \\
\hline 50:40:10 & Diosgenin & No movement & No movement & No movement & 34 \\
\hline \multirow{2}{*}{$\begin{array}{l}\text { n-butanol:acetic } \\
\text { acid:water }\end{array}$} & Digitoxin & 95 & 54 & Moved to SF & 66 \\
\hline & Ouabain & No movement & 06 & No movement & 30 \\
\hline $50: 20: 30$ & Diosgenin & No movement & No movement & No movement & 38 \\
\hline \multirow{3}{*}{$\begin{array}{l}\text { n-butanol:acetic } \\
\text { acid 90:10 }\end{array}$} & Digitoxin & 89 & 52 & 65 & 61 \\
\hline & Ouabain & No movement & 41 & 22 & 25 \\
\hline & Diosgenin & No movement & No movement & No movement & 35 \\
\hline \multirow{3}{*}{$\begin{array}{l}\text { Chloroform: } \\
\text { acetone: ethanol } \\
\text { 50: } 40: 10\end{array}$} & Digitoxin & Moved to SF & Moved to SF & Moved to SF & 64 \\
\hline & Ouabain & No movement & No movement & No movement & 44 \\
\hline & Diosgenin & 36 & 27 & 31 & 59 \\
\hline \multirow{3}{*}{$\begin{array}{l}\text { Chloroform: } \\
\text { ethanol 80: } 20\end{array}$} & Digitoxin & Moved to SF & 96 & 91 & 67 \\
\hline & Ouabain & No movement & 01 & 07 & 34 \\
\hline & Diosgenin & 22 & 14 & 17 & 38 \\
\hline \multirow{3}{*}{$\begin{array}{l}\text { Chloroform: } \\
\text { ethanol 90: } 10\end{array}$} & Digitoxin & 80 & 66 & 50 & 62 \\
\hline & Ouabain & 15 & 33 & 60 & 28 \\
\hline & Diosgenin & 61 & 43 & 57 & 41 \\
\hline
\end{tabular}

hyoscine and strychnine remained at the origin. The reverse was the case with Methanol: chloroform 60:40 except in Irish potato starch layers. With a lesser polar solvent system, Acetone: chloroform 40:60 all alkaloids moved, the movements becoming more appreciable as much lesser polar solvent systems Benzene: acetone: chloroform 20:30: 50 and Pentane: diethylamine 80:20 were used. When the starch layers were impregnated with formamide and and papaverine moved on Guinea corn layers. With Chloroform: petroleum ether 30: 70, all the alkaloids moved on the three starch layers. When alkaloids were tried on acetate plates using non-polar solvent systems, Pentane: diethylamine 80: 20, no separation occurred, but with polar solvent systems, Ethanol: chloroform 10: 90 they moved at different rates. 


\section{Discussion}

The proportion of water used to prepare the slurry varies with different adsorbents. For silica gel, twice the amount of water is used i.e. $30 \mathrm{~g}$ of silica gel is made into slurry with $60 \mathrm{ml}$ of water (ratio 1: 2). This ratio on experimentation with starch did not yield good slurry resulting in uneven layers on the plates. With $50 \mathrm{ml}$ of water to $30 \mathrm{~g}$ of starch, plates with uniform layers were formed. The derivatised starch (acetate) being hydrophobic did not form good suspensions with water, unlike the starch slurry, but formed soaplike suspensions, which did not yield uniform plates. Absolute ethanol was used to obtain good suspensions. The plates were prepared without using any binder as is the case with other adsorbents. Silica gel $G$ contains binder "gypsum". The binder is employed to ensure that a physically stable layer that does not flake is acids should contain water and markedly polar organic solvents may be used as components. When non-polar solvents, petroleum ether and chloroform were used, the amino acids remained at the origin because of poor solubility, likewise when n-butanol was alone. However when polar solvents like water, diethylamine, acetic acid and acetone were added to n-butanol, movement and sharp separations were observed. From Table 1, n-butanol: acetic acid: water (60: 15: 25), n-butanol: acetic acid: water (60:30: 10) and n-butanol: acetone: diethylamine: water (35: 35: 10:20) in that order are considered best for the separation of amino acids on starch plates.

Some steroids, for example digitoxin and oubain, contain a number of hydroxyl (-OH) groups hence are polar and are expected to be separated on starch plates. Diosgenin on the other hand, is relatively non-polar because it contains very few oxygen atoms. When non-

TABLE 3: $\mathrm{HR}_{\mathrm{f}}$ values of 2 Fixed oils on plain and paraffin impregnated starch layers with Silica gel as standard

\begin{tabular}{|c|c|c|c|c|c|}
\hline & & Cassava & Irish potato & Guinea corn & $\begin{array}{l}\text { Silica } \\
\text { gel }\end{array}$ \\
\hline Solvent systems & Spots & $R_{f}(x 100)$ & $R_{f}(x 100)$ & $R_{f}(x 100)$ & $R_{f}(x 100)$ \\
\hline Chloroform: & Castor oil & Moved to SF & Moved to SF & 85 & 80 \\
\hline $\begin{array}{l}\text { petroleum ether } 60 \text { : } \\
40\end{array}$ & Cod liver oil & Moved to SF & Moved to SF & 79 & 75 \\
\hline $\begin{array}{l}\text { Chloroform: } \\
\text { petroleum } \quad \text { ether: }\end{array}$ & Castor oil & Moved to SF & Moved to SF & Moved to SF & $\begin{array}{l}\text { Moved to } \\
\text { SF }\end{array}$ \\
\hline acetone 60: 30: 10 & Cod liver oil & Moved to SF & Moved to SF & Moved to SF & $\begin{array}{l}\text { Moved to } \\
\text { SF }\end{array}$ \\
\hline \multicolumn{6}{|c|}{ Fixed oils with paraffin impregnated starch layers with silica gel as standard. } \\
\hline Chloroform: ethanol & Castor oil & No movement & No movement & No movement & 92 \\
\hline 90: 10 & Cod liver oil & No $m$ & No $m$ & No $n$ & 86 \\
\hline n-butan & Castor oil & 58 & 70 & 41 & 44 \\
\hline $60: 40$ & Cod liver oil & 62 & 65 & 45 & 41 \\
\hline
\end{tabular}

formed ${ }^{(7)}$. Starch is sometimes employed as a binder because of its adhesive properties. As expected the starches formed stable layers without the inclusion of binders.

Starch is a polysaccharide with a combination of maltose units, hence contains many hydrophilic groups and is consequently strongly hydrophilic. The amino acids are markedly hydrophilic and have extremely limited solubility in organic solvents, as such it has been recommended that chromatographic solvent systems for amino polar solvents namely chloroform and petroleum ether was used in the ratios of 50: 50 and 60: 40, diosgenin moved to the solvent front.

Diosgenin and oubain being polar were experimented with the polar solvent systems used for the amino acids. Digitoxin moved almost to the solvent front while oubain remained at the origin. This shows that digitoxin is a more polar constituent than oubain due to the sugar moieties of the two glycosides. Oubain is a monoglycoside while digitoxin is a 
triglycoside ${ }^{(10)}$. Less polar solvents were used to decrease the movement of digitoxin and cause oubain to move. The highly polar components, water and diethylamine were removed so nbutanol and acetic acid 90: 10 was used. From Table 2, oubain moved just slightly on Irish potato and Guinea corn plates and remain at the origin on Cassava plates, but digitoxin moved appreciably. This solvent system can therefore serve as a good separating system for oubain and digitoxin. In other less polar systems employed, chloroform: ethanol 80: 20 and chloroform: acetone: ethanol 50: 40: 10, digitoxin moved up to the solvent front while oubain remained at the origin. More non-polar solvent system chloroform: ethanol 90: 10 resulted in oubain moving slightly.

Castor oil and Cod liver oil, like most fixed oils are soluble in Petroleum ether and Chloroform. Good developments did not occur with fatty acids and fixed oils probably because they are non polar while starch is polar. Fatty acids also contain carboxyl group (- $\mathrm{COOH})$ and thus have very little affinity for starch. A modification to make the stationary phase non polar was carried out by impregnating the starch with liquid paraffin. The starch thus acts as a support for the liquid stationary phase, the system therefore becoming a partition chromatography ${ }^{(11)}$. The stationary phase being non polar, polar solvents were used as the mobile phase, hence with n-butanol and acetic acid $60: 40$, both castor oil and cod liver oil moved in all the systems. The idea of impregnating with paraffin ${ }^{(12)}$ was put forward by Ankwer and Sonanini on silica gel plates and it has proved useful with starch layers.

Alkaloids have varied structures, therefore differ in polarity. Knowledge of the solubility of alkaloids and their salts is of Pharmaceutical importance (10). Not only are alkaloidal substances often administered in solution, but also the differences in solubility between alkaloids and their salts provide methods for the isolation of alkaloids from the plant and their separation from the non-alkaloidal substances also present. While the solubilities of different alkaloids and salts show considerable variation, as might be expected from their extremely varied structure, it is true to say that the free bases are frequently sparingly soluble in polar solvents but soluble in non polar solvents; with salts the reverse is often the case, these being usually soluble in polar solvents but sparingly soluble in organic solvents.

Formamide impregnation having been tried on cellulose layers successfully was also tried on starch layers which are also organic like cellulose and differ very slightly structurally. Formamide impregnated plates being polar required non polar solvents as the mobile phase. Similar solvents as have been used on cellulose were also used.

Acetate plates were used, with both polar and non polar solvent systems. Acetylation can be carried out with agents like acetyl chloride, acetic anhydride or acetic acid. These agents provide the acetyl group which converts the $-\mathrm{OH}$ group of alcohols and acids to acetate esters. Acetylation with acetic anhydride is usually best carried out in pyridine solution, as solvent and catalyst or in the presence of sodium acetate or concentrated sulphuric acid as catalyst (13). Acetylation using pyridine has been successfully carried out with primary and secondary alcohols and with sugars, however, pyridine did not dissolve the starches and acetate was not formed after following the usual acetylation procedure. Acetylation on cellulose to produce cellulose triacetate has been performed with acetic anhydride, acetic acid and concentrated sulphuric acid ${ }^{(14)}$. This method was tried on the starches with success.

In this study, it has been proven that the three starches evaluated with varying solvent systems are suitable adsorbents both in their naturally occurring forms and modified forms. 


\section{Abere et al}

TABLE 4: $\quad \mathrm{HR}_{\mathrm{f}}$ values of 4 Alkaloids on plain and formamide impregnated and acetate starch layers with Silica gel as standard.

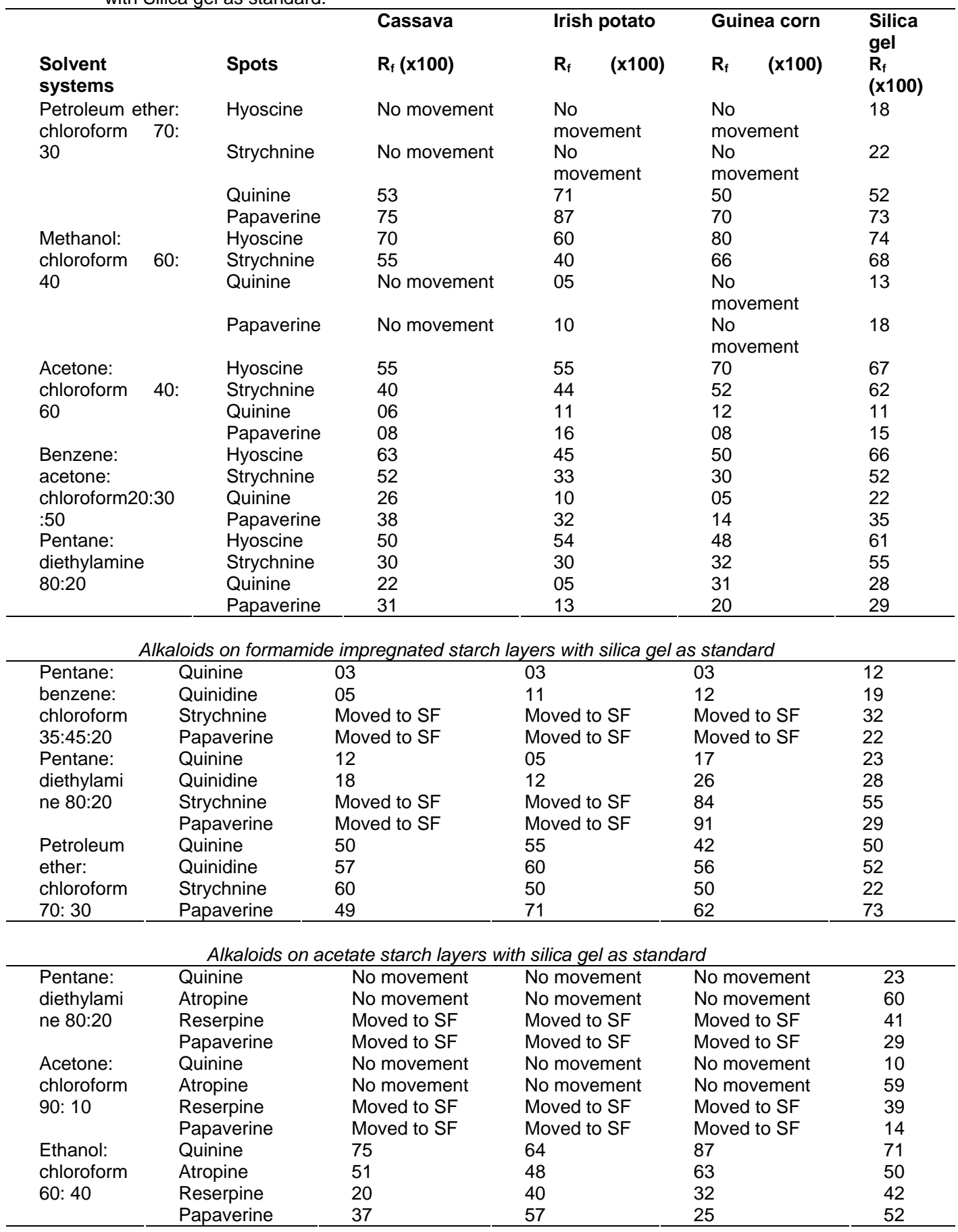




\section{Abere et al}

\section{References}

1. Esezobo S. (1986). Evaluation of Sweet potato starch as a binder and disintergrant for paracetamol tablets. Nig. J. Pharm. Sci. 2(2), 44-51.

2. Iwuagwu M.A. Nwaoshai C.A. and Iseghohi F.E. (1986) Investigations into the binding and disintergrant properties of starches from selected Dioscoteaceae plants. Nig. J. Pharm. Sci. 2(2), 10-22.

3. Desphande A.V. and Panya L.B. (1987). Evaluation of Sorghum starch as a tablet disintergrant and binder. J. Pharm. Pharmacol., 39, 495-496.

4. Mitrevej A. Sinchaipanid A and Faroongsarng D. (1996). Spray-dried rice starch; comparative evaluation of direct compression filters. Drug Dev. Ind. Pharm. 22(7), 587-594.

5. Iwuagwu M.A. and Akerele J.O. (2001). Microbiological suitability of African bitter yam starch. Pak. J. Sci. Ind. Res. 44(4). 227-230.

6. Subhadhirasakul S. Yuenyoungsawad S. Ketyinda W. Phadoongsombut N. and Faroongsarng D. (2001). Study on tablet binding and disintergrant properties of alternative starch prepared from Taro and Sweet potato tubers. Drug Dev. Ind. Pharm. 27(1), 81-87.
7. Oherhorm R.A. (1980) Chromatography: In Remington Pharmaceutical Sciences. $16^{\text {th }}$ edition. Mack Publishing Co. Eastern Pennsylvania. 113-128.

8. Clark E.G.C. (1971). Isolation and identification of drugs. 1st edition. Pharmaceutical Press London. $31-46$

9. Randerath K. (1968). Thin layer chromatography, translated by D.D. Libman. $2^{\text {nd }}$ edition, Verlay Chemie, Academic Press, New York and London. 224.

10. Evans W.C. (2002). Trease and Evans Pharmacognosy. $15^{\text {th }}$ edition. W.B. Saunders, London. 334.

11. Saunders D.L. (1975). Chromatography. $3^{\text {rd }}$ edition, Van Nostrand Reinhold, London. 51-54.

12. Stahl E. (1969). Thin layer Chromatography. $2^{\text {nd }}$ edition, Springer Verlag Berlin. 25-27.

13. Dazeley G.H. (1969). Organic Chemistry. Vol.1. Cambridge University Press. 113-114.

14. Finar I.L. (1973). Organic Chemistry. The fundamental principles. $6^{\text {th }}$ edition. The English Language Book Society and Longman group limited. 89-91. 